\title{
The Differences of the Psychological Status of the Public Between Hubei Provinces and Other Provinces After the COVID-19 Outbreak: a Cross-sectional Study
}

\author{
Na Du ( 13920785057@163.com) \\ The Fourth People's Hospital of Chengdu \\ Yingjie Ouyang \\ the fourth people's hospital of chengdu \\ Yunge Li \\ the fourth people's hospital of chengdu \\ Manxi He \\ the fourth people's hospital of chengdu \\ Ting Geng \\ the fourth people's hospital of chengdu \\ Chunya Li \\ the fourth people's hospital of chengdu \\ Yalan Hu \\ the fourth people's hospital of chengdu \\ Fengyu Liu \\ the fourth people's hospital of chengdu \\ Li Zhang \\ the fourth people's hospital of chengdu \\ Min Zhu \\ the fourth people's hospital of chengdu \\ Lishi Luo \\ the fourth people's hospital of chengdu \\ Juan Huang \\ the fourth people's hospital of chengdu \\ Hongming Wang \\ the fourth people's hospital of chengdu \\ Yong Chen \\ the fourth people's hospital of chengdu \\ Yuchuan Yue \\ the fourth people's hospital of chengdu \\ Maoxiang Xiong \\ the fourth people's hospital of chengdu \\ Keliang Pan \\ the fourth people's hospital of chengdu
}

Research

Keywords: COVID-19, mental health, public, anxiety, depression

Posted Date: August 13th, 2020

DOI: https://doi.org/10.21203/rs.3.rs-56124/v1

License: (9) (1) This work is licensed under a Creative Commons Attribution 4.0 International License. Read Full License 


\section{Abstract}

Background: Assessing the psychological status of public from different areas after the COVID-19 outbreak in China and analyzing the risk factors.

Methods: A cross-sectional survey via online questionnaires was conducted. Occurrence of pressure, anxiety and depression were measured with Perceived Stress Scale (PSS), Generalized Anxiety Scale (GAD-7), and Patient Health Questionnaire-9 (PHQ-9), respectively.

Results: The study included 3300 participants, with 1644 from Hubei and 1656 from outside Hubei. The rates of anxiety and depression among subjects from Hubei were $59.6 \%$ and $52.3 \%$, while the corresponding rates for those outside Hubei were $44.4 \%$ and $46.6 \%$. The scores of PSS, GAD-7, and PHQ-9 in Hubei were significantly higher than those in other areas $(t=3.823,5.860,2.211 ; P<0.05)$. Multiple linear regression analysis indicated that among the participants from Hubei, the higher the educational background, the history of psychosis, the worse their physical health, the COVID-19 infected, isolated, and their families infected with the COVID-19, their mental health was relatively poor. In the participants outside Hubei, the more unstable their marriage status, the worse their physical health, isolated, and their families infected with the COVID-19, the worse their mental health level.

Conclusions: The stress, anxiety, and depression of the public in Hubei are more prominent and the factors that affect their mental health are more complex. When conducting psychological intervention on the general public, we still need to formulate special intervention plans according to the different characteristics of different groups of people to provide them with appropriate crisis intervention services.

\section{Background}

On January 23, Wuhan City, the initial city outbreak of COVID-19, experienced an unprecedented disaster. One week after the outbreak, on January 30, the World Health Organization declared the outbreak a public health emergency of international concern [1]. Considering the high infectivity of COVID-19, the Wuhan municipal government immediately suspended all public transportation to prevent further transmission during the Spring Festival, which was then imitated by several other neighboring cities [2]. At the same time, however, the measures taken by the government to seal the city and the lockdown inevitably caused negative impacts on the life of the public.

This epidemic may become a significant stressor in people's lives, and exposure to such emergencies may cause many psychological and social problems, including some common mental disorders [3]. Relevant research shows that mental health issues cluster around the following themes: anxiety-based symptoms including those of acute stress disorder and post-traumatic stress disorder; grief reactions and depression; generalized distress including increased stress levels and sleep disruption [4]. While 2003, the severe acute respiratory syndrome (SARS) epidemic occurred in Guangdong Province was also a public health emergency. It is one of the well-known infectious diseases with global influence and eventually affected more than 29 countries, resulting in 8,422 cases and 916 deaths worldwide [5]. China as one of the most severely affected countries suffered from an enormous strike. Due to the high infectious potential and mortality rate of SARS, the epidemic led to panic in the affected countries [6]. Studies conducted a corresponding investigation on the mental state of the public after the outbreak of SARS and found that a high level of distress due to SARS was documented in the community [7]. Meanwhile, Lau et al found that the SARS epidemic caused tremendous damage to the public and many individuals exhibited post-traumatic stress symptoms [8]. It was reported that mood disorders were common in the general population during the SARS epidemic [9]. It has been found that even at a relatively low level of spread among the population, the SARS outbreak still had a significant psychological impact, as well as emotional difficulties most frequently reported problems [10].

Generally speaking, after a major disaster or emergency, the psychological conditions of the public may change to different extents. After the outbreak of COVID-19, the latest relevant reports have pointed out that, the epidemic has caused a parallel epidemic of fear, anxiety, and depression [11], similar results were found in Duan and Zhu's research which implied that an increase of psychological problems during this epidemic was noted [12]. Although they conducted a survey on the psychological situation of the corresponding population in time after the outbreak, relevant studies did not specifically analyze the psychological differences between the population in different areas with different severity. Besides, little is known about which factor may be associated with the appearance of the negative change during the epidemic. Therefore, the purpose of this study is to make a comparative analysis of the people who have lived in Hubei Province and the people in other provinces to find out what differences exist in the psychological impact during the epidemic and to discover the differences of their opinion on the epidemic. Meanwhile, through this study, it is expected to find relevant influencing factors that may cause psychological problems during the outbreak. Only by grasping the characteristics of their psychological changes and what are the help they mostly wanted, can we further summarize the characteristics of people's psychological reactions under public health emergencies, and generate future psychological intervention strategies according to the psychological reaction characteristics of people under different epidemic conditions.

\section{Methods}




\section{Participants}

We conducted this cross-sectional study at the time when WHO announced the COVID-19 as a Public Health Emergency of International Concern. The whole survey persisted nearly 3 weeks until the end of February. Due to the impact of the epidemic, we couldn't conduct face-toface assessment on participants. Therefore, we adopt the most secure and effective method of network self-assessment. On one hand, we can spread the questionnaire to a greater extent, on the other hand, we can quickly retrieve the questionnaire and give timely feedback to all the participants, and provide them with different degrees of psychological support online (including the different link of relaxation training according to their scores / the hotline number of psychological assistance in our hospital).

Only adults (aged $\geq 18$ years) of Chinese nationality who were able to provide verbal informed consent were recruited in the study using convenience and snowball sampling methods. The online survey was initially distributed to community residents who were encouraged to pass it on to others. A total of 3680 participants were recruited. Among them, 380 were excluded due to incomplete survey responses, or they are health workers who worked at the frontline. (Due to their endurance of above-normality stressors, it may influence the overall results). The recuperation rate of the questionnaire was $89.7 \%$. All responses were anonymous. The survey questionnaire included questions about demographic characteristics, the experience of the COVID-19 epidemic, related opinions on this epidemic, self-perceived health status, Perceived Stress Scale (PSS), Generalized Anxiety Scale (GAD-7), and 9-item patient health questionnaire (PHQ-9). The study was approved by the hospital ethics committee. In this survey, participants were divided into 2 different groups: One survey involved 1644 adult residents who have lived in Hubei since the COVID-19 outbreak, excluding those who have left Hubei since the outbreak; the other survey involved adults nationwide outside Hubei, with a sample size of 1656 .

\section{Measures}

\subsection{The Questionnaire of demographic characteristics, related experience, and opinion on COVID-19}

The demographic background survey includes gender, age, marital status, educational level, and employment status. Experience related to the epidemic includes whether they have been infected with COVID-19, whether their family members have been infected with COVID-19, and whether they have been quarantined. Opinions on the epidemic include the most demanding help, the most dominant source of nervousness, and the most dominant source of sadness during the epidemic.

\subsection{Self-perceived health status}

The rating is based on a 5-point scale: 1 point is that the physical condition is very good; 2 points is that the physical condition is good; 3 points is that the physical condition is average; 4 points is that the physical condition is poor; 5 points is that the physical condition is very poor.

\subsection{Perceived Stress Scale (PSS) [13]}

It is a self-assessment scale developed by Cohen et al. to assess the degree of stress an individual feels in the past month. There are 10 items in the PSS-10 version used in this study, including 6 items with negative description (item 1, 2, 3, 6, 9, 10) and 4 items with positive description (item $4,5,7,8$ ). Each item is scored on a 5 -grade scale ranges from 0 to 4 . The total score is the sum of the scores for all items. The higher the score, the greater pressure the individual experiences. The Chinese version of the scale has been proved to have good reliability and validity [14].

\subsection{Generalized Anxiety Scale (GAD-7) [15]}

The GAD-7 consists of seven items, each item scores by 0-3 points, the total score range is 0-21 points. Among them, 0-4 points are no anxiety; 5-9 points are mild anxiety; 10-14 points are moderate anxiety; 15-21 points are severe anxiety. The scale has been used in China for many years and has been proved to have good reliability and validity, which can help to distinguish the severity of anxiety [15].

\subsection{9-item patient health questionnaire (PHQ-9) [16]}

It consists of 9 items, each item scores 0-3 points, the total score range is 0-27 points. Among them, 0-4 points are no depression; 5-9 points are mild depression; 10-14 points are moderate depression; 15-19 points are moderate and severe depression; 20-27 points are severe depression. The scale has been used in China for many years and has been proved to have good reliability and validity, which helps to distinguish the severity of depression [17].

\subsection{Statistical analysis}


For each scale, the scores were summed. The mean scores were then used for comparison among groups using the T-test and analysis of variance (ANOVA) statistics. First, the influencing factors that may be related to PSS, GAD-7, and PHQ-9 were screened out by one-way ANOVA, and then these factors were incorporated into the multiple linear region model, and the relevant influencing factors of the total scores of the above three scales were analyzed respectively. All data were analyzed using Statistical Package for Social Sciences (SPSS) version 21.0. P-values of less than 0.05 were considered statistically significant (2-sided tests).

\section{Results}

\section{General information}

\subsection{Demographic and epidemic related information}

The survey involved 3300 participants, including 880 men (26.67\%) and 2420 women (73.33\% ). Among all, 1644 were from Hubei Province, with an average age of $44.20 \pm 11.33$ years old; 1656 participants were from outside Hubei (involves 15 provinces), with an average age of $39.18 \pm 15.69$ years old (there was no significant difference in average age between the two groups: $t=1.063, p=0.288$ ). For participants from Hubei, 52 had suffered from psychiatric diseases, including 12 with depression, 8 with bipolar disorder, and 32 with anxiety. For those outside Hubei, 68 had suffered from psychiatric diseases, including 4 with drug-induced mental disorder, 16 with depression, and 48 with anxiety disorders. Table 1 presents the remaining demographic information.

\subsection{Opinions on the epidemic situation}

Opinions on the epidemic situation of the participants were obtained through the questionnaire. The most needed help of the participants in Hubei during the epidemic was: improved medical conditions (996 people, accounting for $60.6 \%$ ), reasonable living arrangements (264 people, $16.1 \%)$; psychological support (176 people, $10.7 \%$ ); no need for help (116 people, $7.1 \%$ ); cooperation of the patients (56 people, $3.4 \%$ ); and communications with their families (36 people, $2.2 \%$ ). While the most needed help of the participants outside Hubei was: improved medical conditions (696 people, $42 \%$ ); reasonable living arrangements (264 people, $15.9 \%$ ); psychological support (260 people, $15.7 \%$ ); no need for help (160 people, $9.7 \%$ ); cooperation of the patients (168 people, $10.1 \%)$; and communications with their families (108 people, $6.5 \%)$.

Participants from Hubei ranked the top three stressful situations during the epidemic (multiple choices): infected people may not be quarantined (1272 people), shortage of protective supplies (1091 people), and infected people did not take protective measures (853 people). For participants outside Hubei, the ranking was: infected person may not be quarantined (867 people), increase of newly confirmed cases (832 people), and shortage of protective supplies (810 people).

The top three distressing situations for participants in Hubei during the epidemic were (multiple choices): helpless patients (1254 people), helpless medical staff (1232 people), and innocent people (869 people). For participants outside Hubei, the ranking changed into: helpless medical staff (1136 people), helpless patients (1008 people), and innocent people (945people).

\section{Analysis of scores of PSS, GAD-7, and PHQ-9 in different regions}

The scores of PSS, GAD-7, and PHQ-9 in Hubei and outside Hubei are shown in Table2. There are significant differences in the three scales between different regions $(t=3.823, p<0.001 ; t=5.860, p<0.001 ; t=2.211, p=0.027$, respectively). The results suggest that participants in Hubei suffered more pressure, anxiety, and depressive symptoms than those outside Hubei.

$59.6 \%$ (980/1644) subjects from Hubei scored above the GAD-7 cut-off point, indicating widespread anxiety among the participants. While the corresponding rate of subjects outside Hubei was $44.4 \%$ (735/1656). The distribution of severity were shown in Figure 1 a.

The PHQ-9, used to assess depression levels, showed that 52.3\% (860/1644) subjects from Hubei had a standardized score of $\geq 5$, deemed as having depression symptoms, and the corresponding rate of subjects outside Hubei was $46.6 \%$ (772/1656). The distribution of severity were shown in Figure 1b.

\section{Single factor analysis of the influencing factors of total scores of PSS, GAD-7, and PHQ-9}

After grouping based on different demographic characteristics and epidemic information, the scores of the PSS among the participants were compared. The results showed that there were statistically significant differences $(P<0.05)$ in terms of educational background, previous mental illness, self-perceived physical health, infection of the COVID-19, quarantine, and whether their family members were quarantined. Comparison of the GAD-7 scores showed that the differences between different genders, different educational background, previous mental illness, self-perceived physical health, whether they were infected with COVID-19, and whether they were isolated were statistically significant $(\mathrm{P}<0.05)$. And the PHQ-9 comparison results showed that there were statistically significant differences in different educational background, 
previous mental illness, self-perceived physical health, whether they were infected with COVID-19, and whether they were isolated in different groups $(\mathrm{P}<0.05)$. See Table 3 for details.

Similarly, for participants outside Hubei, the different scores of the PSS and GAD-7 showed statistically significant differences (P < 0.05 ). And the comparison of the PHQ-9 scores showed that there were statistically significant differences in self-perceived physical health and whether they were quarantined $(P<0.05)$. See Table 4 for details.

\section{Multiple stepwise regression analysis of influencing factors of total scores of PSS, GAD-7, and PHQ-9}

\subsection{Multiple stepwise regression analysis on the influencing factors of total scores of PSS, GAD-7, and PHQ-9 in Hubei}

Taking the total score of the PSS scale as the dependent variable and factors with statistical significance in univariate analysis as independent variables. Table 3 shows the assignment of each factor. The analysis results suggest that the main factors that influencing the PSS score are educational background, history of psychiatric related diseases, physical health, whether they were infected by the COVID-19, whether they were quarantined, and whether their family members were infected by the COVID-19 (P<0.05), as shown in Table 5. The integrity test was performed on the regression equation $F(6,1637)=116.871(P<0.001)$, and it shows that the fitted multiple linear stepwise regression equation has a statistical significance. After evaluating the regression equation model, the multiple correlation coefficient $\mathrm{R}=$ 0.797 and the coefficient of determination $=0.634$ were calculated. These six independent variables can effectively explain the $63.4 \%$ variance of the stress level suffered by the participants.

When taking the total score of GAD-7 as the dependent variable and the factors with statistical significance in the univariate analysis as independent variables for analysis. See Table 3 for the assignment of each factor. It suggests that the main factors affecting the score of the patient's anxiety scale are gender, history of psychiatric related diseases, physical health, whether they were infected with COVID-19, and whether they were quarantined $(P<0.05)$, see Table 5 for details. The integrity test performed on the regression equation $F(6,1637)=89.729$ $(P<0.001)$ shows that the fitted multiple linear stepwise regression equation has statistical significance. After evaluating the regression equation model, multiple correlation coefficient $\mathrm{R}=0.765$ and the coefficient of determination $=0.565$ were calculated. Therefore, these five independent variables can effectively explain the $56.5 \%$ variance of anxiety.

If take the total score of PHQ-9 as the dependent variable and the factors with statistical significance in the univariate analysis as independent variables. The assignment of each factor is shown in Table 3. The results suggest that the main factors affecting the patients' depression scale scores are educational background, physical health, and whether they were quarantined $(P<0.05)$, see Table 5 for details. The integrity test for the regression equation $F(4,1639)=21.395(P<0.001)$ shows that the fitted multiple linear stepwise regression equation has statistical significance. After evaluating the regression equation model, the multiple correlation coefficient $\mathrm{R}=0.678$ and the coefficient of determination $=0.438$ were calculated. Therefore, these three independent variables can effectively explain the $43.8 \%$ variance of anxiety of anxiety.

\subsection{Multiple stepwise regression analysis on the influencing factors of the total scores of PSS, GAD-7, and PHQ-9 of the participants outside Hubei}

Likely, taking the total score of PSS as the dependent variable and the factors with statistical significance in the univariate analysis as independent variables. The analysis results imply that the main factors affecting the score of the patient's stress scale are marital status, physical health, and whether they were quarantined $(P<0.05)$, as shown in Table 6 . The integrity test was performed on the regression equation $F(3,1652)=46.163(P<0.001)$ indicating that the fitted multiple linear stepwise regression equation has statistical significance. After evaluating the regression equation model, multiple correlation coefficient $R=0.731$ and coefficient of determination= 0.534 were calculated. Therefore, these three independent variables can effectively explain the $53.4 \%$ variance of anxiety.

When taking the GAD-7 scale total score as the dependent variable and the factors with statistical significance in the univariate analysis as independent variables for analysis. Analysis results suggest that the main factors affecting the score of patients' anxiety scale are physical health, whether they were quarantined, and whether their family members were infected with COVID-19 (P< 0.05$)$, see Table 6 for details. The integrity test $F(4,1651)=100.047(P<0.001)$ performed on the regression equation indicates statistical significance in the fitted multiple linear stepwise regression equation. After evaluating the regression equation model, the multiple correlation coefficient $\mathrm{R}=0.831$ and the coefficient of determination $=0.690$ were calculated. These three independent variables can effectively explain $69.0 \%$ of the variance of anxiety.

If take the total score of the PHQ-9 scale as the dependent variable and the factors with statistical significance in the univariate analysis as independent variables. Analysis results show that the main factors affecting the scores of depression scale of patients are physical health and isolation $(P<0.05)$, see Table 6 . The integrity test was conducted on the regression equation $F(2,1653)=25.235(P<0.001)$, it shows 
that the fitted multiple linear stepwise regression equation has statistical significance. After evaluating the regression equation model, the multiple correlation coefficient $\mathrm{R}=0.708$ and the coefficient of determination $=0.481$ were calculated. Thus, these two independent variables can effectively explain the $48.1 \%$ variance of anxiety.

\section{Discussion}

This is the first study to investigate the mental health and their influencing factors of public from areas with severe epidemic three weeks after the occurrence of COVID-19, and the data was compared with those from areas with mild epidemic. We investigated 3300 participants in total, and found symptoms of anxiety, depression and pressure to be common in general public, though the severity of symptoms of the public in Hubei was greater.

After investigation, we found that the public believed that improvements in medical conditions would be their greatest help. Since our investigation was conducted in the early stage of the outbreak, the number of new diagnosis showed a blow-up tendency, while the number of infectious disease specialist hospital in China was very limited. The imbalance caused a shortage of hospital beds, many patients with mild diseases were encouraged to be quarantined at home, which inevitably increased their pressure. The second most needed help is reasonable arrangement of daily life. As a result of the epidemic, many people's life were disrupted. The original seven-day vacation was continuously extended. A survey found that people's daily life changed during the epidemic, which was getting up later and earlier dinner, it made many people become "night owls" [18]. These changes in schedules brought many people with discomfort in life, which further aggravated their bad mood. Besides, both groups thought that psychological support was very necessary. Because lifestyle changes can make people feel anxious [19], they felt that if they could receive appropriate psychological support during this period, they could have part of their psychological burden relieved. Through the above results, the government should expand medical resources in the first place in the face of severe outbreaks; and secondly, it is necessary to establish a psychological support intervention system for the public as soon as possible and provide reasonable life guidance to the public [20], so as to reduce psychological discomfort of the public. .

We found that the participants from Hubei and those outside Hubei thought the lack of quarantine of potential infections and the shortage of protective supplies made them nervous. Because COVID-19 is highly contagious, people are most worried about whether they will be infected. Therefore, if the isolation measures were not implemented properly, it would inevitably make people feel anxious. As Shimura et al. found, after confirming that the virus can be passed from person to person, people's anxiety-related behaviors increased significantly [21]. For people outside Hubei, they believe that the increasing number of confirmed cases makes them feel nervous. Due to the rapid development of the new media nowadays, the public is able to receive information much quicker. Since China has fully learned the experience of SARS prevention and control, after the outbreak, Chine made the epidemic related information accessible and transparent at the very beginning, such that we can see the sharp increase in the number of newly diagnosed patients every day. On the one hand, this could inform the public about the severity of the outbreak and alert them such that they can take active protective measures to minimize the chance of infection. On the other hand, it represents the respect of the Chinese government for people worldwide. By reporting the latest progress of the outbreak to the world on a regular basis, the Chinese government provided a reference for the prevention and control measures to other countries. However, this inevitably brought certain psychological pressure on the public, and the pressure will be more prominent to the non-infected people. This suggests that we need to continue to strengthen the guidance of anxiety relief for this part of the public. .

All participants believed that helpless infected people, helpless medical staff, and innocent people were their dominant source of sadness. Due to the impact of the epidemic, many people were isolated and could not go home during the holidays, and the shortage of medical staff also made medical resources in a dilemma. These factors made people feel helpless. In addition, there were also numerous reports about infections of medical staff who were on the front line [22], which undoubtedly increased the sadness of the public. We realize that when providing psychological support to the public, we must fully consider their pity for infected people and medical staff, and increase their confidence from this aspect, encouraging them to pay more attention to positive reports about the epidemic rather than sinking into negative news repeatedly.

This study found that the scores of the stress, anxiety, and depression scales of the participants in Hubei were significantly higher than those outside Hubei. Although there have been reports of a mild stressful impact on people in non-epidemic areas, that these people did not feel helpless obviously [23]. A review of the published literature on COVID-19 suggests that the common psychological reactions of the public after the outbreak include anxiety, depression, and self-reported stress [24]. Although the above-mentioned reactions are common in the public, there is no comparative analysis of the psychological stress reaction between the public in the epidemic area and the public in the nonepidemic area so far. Our investigation further confirmed that people in the severe epidemic area underwent more intense pressure, and their anxiety and depression symptoms are more prominent. This can be fully explained by the detection rates of anxiety and depression symptoms in different areas. Thus, when we encounter similar outbreaks in the future, the focus of psychological intervention still needs to be placed in the origin of the epidemic first. As the Chinese government gradually realized this, it gradually increased the proportion of 
psychiatrists and psychotherapists when dispatching different batches of medical teams to help Hubei, in order to be able to carry out psychological crisis intervention for the public in Hubei as soon as possible.

Through multiple regression analysis, we found that for participants in Hubei, the higher their education level, who had suffered from psychiatric diseases in the past, who thought that their health level was worse, who had been infected with COVID-19, who had been isolated and whose family members had been infected with COVID-19, the higher their stress level was. Women, who have suffered from mental diseases in the past, who think that their physical health are worse, who have been infected with COVID-19, who have been isolated, have more prominent anxiety symptoms. In addition, the higher the education level, the lower the self-perceived physical health and the experience of being isolated, the more severe the depressive symptoms. It can be seen that previous psychiatric diseases have greater impacts on the psychological state of people in the epidemic area. The outbreak of this epidemic is undoubtedly a crisis for these people, they tend to be more nervous and worried. This crisis affected their medical treatment to a certain extent and might make their mental symptoms worse [11]. This undoubtedly reminds us that we need to carry out crisis intervention services as early as possible and provide alternative medical treatment programs for this group to avoid their original disease recurrence and increase the social burden [25]. Besides, we found that those with poor self-perceived physical conditions endured more stress, anxiety, and depression. One reasonable explanation is that these people were not optimistic about their health , they were more worried that their bodies can not resist the virus, which could make them be more sensitive than ordinary people. As Wang et al. found, being satisfied with their own health can become a protective factor for people's mental health during the epidemic [26]. Those who had been quarantined due to the epidemic also showed a relatively poor mental health status. This result is similar to many reports [27-28]. Due to the social attributes of human beings, all humans are at risk of psychological harm when keeping in isolation [29], which reminds us that we should pay more attention to the mental health of those who have experienced isolation and provide them with timely psychological intervention. Furthermore, we found that women are more likely to experience anxiety than men, which is consistent with the report of Wang et al [30], indicating that women's emotional experience is more prominent and more worried when facing the epidemic. In this study, we were surprised to find that the higher the education level, the heavier the pressure and depression. Relatively speaking, lower educational level becomes a protective factor when facing the epidemic. The reason for this phenomenon may be that the higher the education level, the greater the amount of information they can receive about the epidemic. The overloaded information can put them in a relatively high-pressure environment [31]. The more delicate, the attitude towards things will be more serious. So in the face of such an outbreak, they tend to express more concerns, which undoubtedly becomes a burden on their emotions [32]..

Unlike participants in Hubei, for those outside Hubei, the more unstable their marital status, the worse their self-perceived health status, and the more pressure they felt when they were isolated. People with poor self-perceived health, quarantine experience, or their family members had been infected with COVID-19 expressed more anxiety symptoms. Besides, those with worse poor self-perceived health and quarantine experience exhibited more depressive symptoms. For people outside Hubei, there are relatively few influencing factors relating to the mental health status after the outbreak, while both groups had screened out similar factors, that is, they thought that their self-perceived health was poor, the mental health of the people who have been isolated was more prominent in stress, anxiety and depression symptoms. Besides, for participants outside Hubei, we found for the first time that the unmarried state became a protective factor for mental health, while the divorced or widowed had a significantly increased sense of stress. The reason for this conclusion may be that unmarried people have relatively few concerns in their lives. If they were quarantined, they may not need to worry about spreading the virus to their spouses or children, so there is no obvious pressure. Although for divorced and widowed people, although they may also be single, they have experienced the companionship of their spouses. In the face of the epidemic, especially when they need to be isolated at home, they may be more vulnerable to loneliness. After all, good family and social support will make people feel at ease [33].

\section{Conclusion}

Through this investigation, we found that the participants in Hubei Province and those outside Hubei had similar views on the epidemic situation. However, the mental health level of the former is significantly worse than that of the latter, and the related factors that affecting the former's mental health are more than the latter, with some unique influencing factors were screened out in both groups. Therefore, when conducting psychological intervention on the general public, we still need to formulate special intervention plans according to the different characteristics of different groups of people to provide them with appropriate crisis intervention services in a targeted manner, such that our services can play the best effect.

\section{Declarations}

Ethics approval and consent to participate: The research has got the institutional review board (IRB) approval of the Fourth People's Hospitarl of Chengdu, and all the subjects were recruited in the principle of voluntary.

Consent for publication: Not applicable. 
Availability of data and materials: The datasets used and/or analysed during the current study are available from the corresponding author on reasonable request.

Competing interests: The authors declare that they have no competing interests.

Funding: Not applicable

Authors' contributions: D.N.: conception and design; D.N., OY.Y.J., L.YG., G.T., L.CY., H.YL., L.FY., Z.L., Z.M., L.LS.and H.J.: conduction; D.N., L.YG and G.T.: statistical analysis; L.YG., W. HM., H. MX., Y. YC., C. Y., X. MX. and P. KL.: administrative, technical, or material support; D.N.: drafting of the manuscript; and OY.Y.J.: critical revision of the manuscript for important intellectual content. All authors read and approved the final paper.

Acknowledgements: We sincerely thank all the healthcare workers who took part in our survey for their assistance in this study.

\section{References}

1.World Health Organization. Statement on the second meeting of the International Health Regulations (2005) Emergency Committee regarding the outbreak of novel coronavirus (2019-nCoV)(2020). Https://www.who.int/news-room/detail/30-01-2020-statement-onthe-secondmeeting-of-the-international-health-regulations-(2005)-emergency-committee-regarding-the-outbreak-of-novel-coronavirus-(2019-ncov) (accessed February 4, 2020)

2. Xu, J., Chen, Y., Chen, H., \& Cao, B: 2019 novel coronavirus outbreak: a quiz or final exam?. Frontiers of medicine 2020; 14(2): $225-228$. https://doi.org/10.1007/s11684-020-0753-1

3.van Ommeren, M., Saxena, S., \& Saraceno, B: Mental and social health during and after acute emergencies: emerging consensus?. Bulletin of the World Health Organization 2005; 83(1): 71-76.

4. Norris F.H. : Psychological consequences of disasters. PTSD Research Quarterly2002; 13 (2):1-7.

5. Leong, H. N., Earnest, A., Lim, H. H., Chin, C. F., Tan, C., Puhaindran, M. E., Tan, A., Chen, M. I., \& Leo, Y. S.: SARS in Singapore--predictors of disease severity. Annals of the Academy of Medicine 2006; Singapore, 35(5): 326-331.

6. Chong, M. Y., Wang, W. C., Hsieh, W. C., Lee, C. Y., Chiu, N. M., Yeh, W. C., Huang, O. L., Wen, J. K., \& Chen, C. L.: Psychological impact of severe acute respiratory syndrome on health workers in a tertiary hospital. The British journal of psychiatry : the journal of mental science 2004; 185: 127-133. https://doi.org/10.1192/bjp.185.2.127

7. Lau, J. T., Yang, X., Pang, E., Tsui, H. Y., Wong, E., \& Wing, Y. K.: SARS-related perceptions in Hong Kong. Emerging infectious diseases 2005; 11(3): 417-424. https://doi.org/10.3201/eid1103.040675

8. Lau, J. T., Yang, X., Tsui, H. Y., Pang, E., \& Wing, Y. K.: Positive mental health-related impacts of the SARS epidemic on the general public in Hong Kong and their associations with other negative impacts. The Journal of infection 2006; 53(2): $114-124$. https://doi.org/10.1016/j.jinf.2005.10.019

9. Lee S.: Mental health issues in the post SARS era. In: Joint HKU/CUHK/CPU academic seminar on 'After SARS: education and research agenda for the future' July 2, 2003. Hong Kong Convention and Exhibition Centre (HKCEC); 2003.

10. Blendon, R. J., Benson, J. M., DesRoches, C. M., Raleigh, E., \& Taylor-Clark, K.: The public's response to severe acute respiratory syndrome in Toronto and the United States. Clinical infectious diseases : an official publication of the Infectious Diseases Society of America 2004; 38(7): 925-931. https://doi.org/10.1086/382355.

11. Yao, H., Chen, J. H., \& Xu, Y. F.: Patients with mental health disorders in the COVID-19 epidemic. The lancet. Psychiatry $2020 ; 7(4)$ : e21. https://doi.org/10.1016/S2215-0366(20)30090-0

12. Duan, L., \& Zhu, G.: Psychological interventions for people affected by the COVID-19 epidemic. The lancet. Psychiatry2020; 7(4): 300302. https://doi.org/10.1016/S2215-0366(20)30073-0

13. Cohen, S., Kamarck, T., \& Mermelstein, R.: A global measure of perceived stress. Journal of health and social behavior 1983; 24(4): 385396.

14. Leung, D. Y., Lam, T. H., \& Chan, S. S.: Three versions of Perceived Stress Scale: validation in a sample of Chinese cardiac patients who smoke. BMC public health 2020; 10: 513. https://doi.org/10.1186/1471-2458-10-513

15.He, X.X., Li, C.B., Qian, J., et al.: Reliability and validity of a generalized anxiety disorder scale in general hospital outpatients. Shanghai archives of psychiatry 2010;22(4): 200-203 
16. Martin, A., Rief, W., Klaiberg, A., \& Braehler, E.: Validity of the Brief Patient Health Questionnaire Mood Scale (PHQ-9) in the general population. General hospital psychiatry 2006; 28(1): 71-77. https://doi.org/10.1016/j.genhosppsych.2005.07.003

17. Wang, W., Bian, Q., Zhao, Y., Li, X., Wang, W., Du, J., Zhang, G., Zhou, Q., \& Zhao, M.: Reliability and validity of the Chinese version of the Patient Health Questionnaire (PHQ-9) in the general population. General hospital psychiatry 2014; 36(5): 539-544. https://doi.org/10.1016/j.genhosppsych.2014.05.021

18. https://www.sohu.com/a/391823920_267106

19. Usher, K., Bhullar, N., \& Jackson, D.: Life in the pandemic: Social isolation and mental health. Journal of clinical nursing 2020. 10.1111/jocn.15290. Advance online publication. https://doi.org/10.1111/jocn.15290.

20. Dong, L., \& Bouey, J. : Public Mental Health Crisis during COVID-19 Pandemic, China. Emerging infectious diseases 2020; 26(7): 16161618. https://doi.org/10.3201/eid2607.200407

21. Shigemura, J., Ursano, R. J., Morganstein, J. C., Kurosawa, M., \& Benedek, D. M.: Public responses to the novel 2019 coronavirus (2019$\mathrm{nCoV}$ ) in Japan: Mental health consequences and target populations. Psychiatry and clinical neurosciences 2020; 74(4): $281-282$. https://doi.org/10.1111/pcn.12988

22. https://www.guancha.cn/politics/2020_03_06_540260.shtml

23. Zhang, Y., \& Ma, Z. F.: Impact of the COVID-19 Pandemic on Mental Health and Quality of Life among Local Residents in Liaoning Province, China: A Cross-Sectional Study. International journal of environmental research and public health $2020 ; 17(7)$ : 2381. https://doi.org/10.3390/ijerph17072381

24. Rajkumar, R.P.: COVID-19 and mental health: A review of the existing literature. Asian J Psychiatr 2020; 52:102066. doi: 10.1016/j.ajp.2020.102066.

25. Zhang, J., Wu, W., Zhao, Z., Zhang, W.: Recommended psychological crisis intervention response to the 2019 novel coronavirus pneumonia outbreak in China: a model of West China Hospital. Precision Clinical Medicine 2020. https://doi.org/10.1093/pcmedi/pbaa006

26. Wang, C., Pan, R., Wan, X., Tan, Y., Xu, L., Mclntyre, R. S., Choo, F. N., Tran, B., Ho, R., Sharma, V. K., \& Ho, C.: A longitudinal study on the mental health of general population during the COVID-19 epidemic in China. Brain, behavior, and immunity 2020; 87: 40-48. https://doi.org/10.1016/j.bbi.2020.04.028

27. Zandifar, A., \& Badrfam, R.: Iranian mental health during the COVID-19 epidemic. Asian journal of psychiatry 2020; 51: 101990. Advance online publication. https://doi.org/10.1016/j.ajp.2020.101990

28. Liu, X., Kakade, M., Fuller, C. J., Fan, B., Fang, Y., Kong, J., Guan, Z., \& Wu, P.: Depression after exposure to stressful events: lessons learned from the severe acute respiratory syndrome epidemic. Comprehensive psychiatry 2012; 53(1): 15-23.

https://doi.org/10.1016/j.comppsych.2011.02.003

29.Wilson, S., McCloughen, A., Parr, J., \& Jackson, D.: "If you are homeless you are welcome here": Social obligations for the homeless and socially disadvantaged. Journal of clinical nursing 2019; 28(15-16): 2721-2723. https://doi.org/10.1111/jocn.14851

30. Wang, C., Pan, R., Wan, X., Tan, Y., Xu, L., Ho, C. S., \& Ho, R. C.: Immediate Psychological Responses and Associated Factors during the Initial Stage of the 2019 Coronavirus Disease (COVID-19) Epidemic among the General Population in China. International journal of environmental research and public health 2020; 17(5): 1729. https://doi.org/10.3390/ijerph17051729

31. Ho, C. S., Chee, C. Y., \& Ho, R. C.: Mental Health Strategies to Combat the Psychological Impact of COVID-19 Beyond Paranoia and Panic. Annals of the Academy of Medicine 2020; Singapore, 49(3): 155-160.

32. Huang, Y., \& Zhao, N.: Generalized anxiety disorder, depressive symptoms and sleep quality during COVID-19 outbreak in China: a webbased cross-sectional survey. Psychiatry research 2020; 288: 112954. https://doi.org/10.1016/j.psychres.2020.112954

33. Maunder, R., Hunter, J., Vincent, L., Bennett, J., Peladeau, N., Leszcz, M., Sadavoy, J., Verhaeghe, L. M., Steinberg, R., \& Mazzulli, T.: The immediate psychological and occupational impact of the 2003 SARS outbreak in a teaching hospital. CMAJ : Canadian Medical Association journal 2003; 168(10): 1245-1251.

\section{Tables}

Table 1. Demographic and epidemic information distribution of participants in Hubei Province and other provinces 


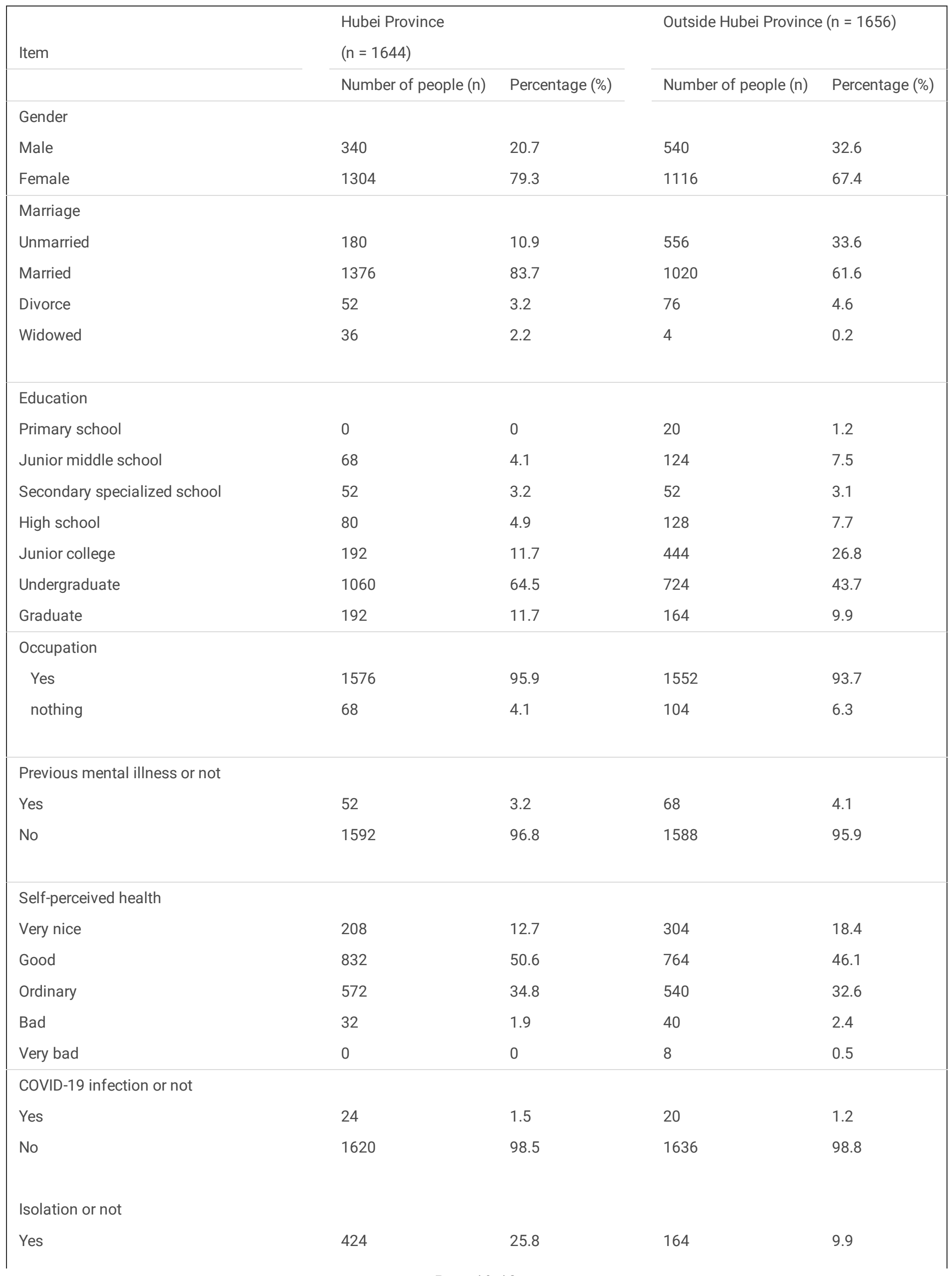


Is the family infected with the COVID-19

Yes

No

148

9.0

20

1.2

1496

91.0

1636

98.8

Table 2. Comparative analysis of PSS, GAD-7, and PHQ-9 of participants in Hubei Province and other provinces

\begin{tabular}{|lllll|}
\hline & $\begin{array}{l}\text { Hubei } \\
(\mathrm{N}=1644)\end{array}$ & $\begin{array}{l}\text { Outside Hubei } \\
(\mathrm{N}=1656)\end{array}$ & T value & P value \\
\hline PSS & $16.21 \pm 7.17$ & $14.33 \pm 6.99$ & 3.823 & $<0.001$ \\
\hline GAD-7 & $7.28 \pm 6.02$ & $5.01 \pm 5.08$ & 5.860 & $<0.001$ \\
\hline PHQ-9 & $6.50 \pm 5.49$ & $5.62 \pm 5.94$ & 2.211 & 0.027 \\
\hline
\end{tabular}

Table 3. Single factor analysis of total scores of PSS, GAD-7, and PHQ-9 in Hubei Province 


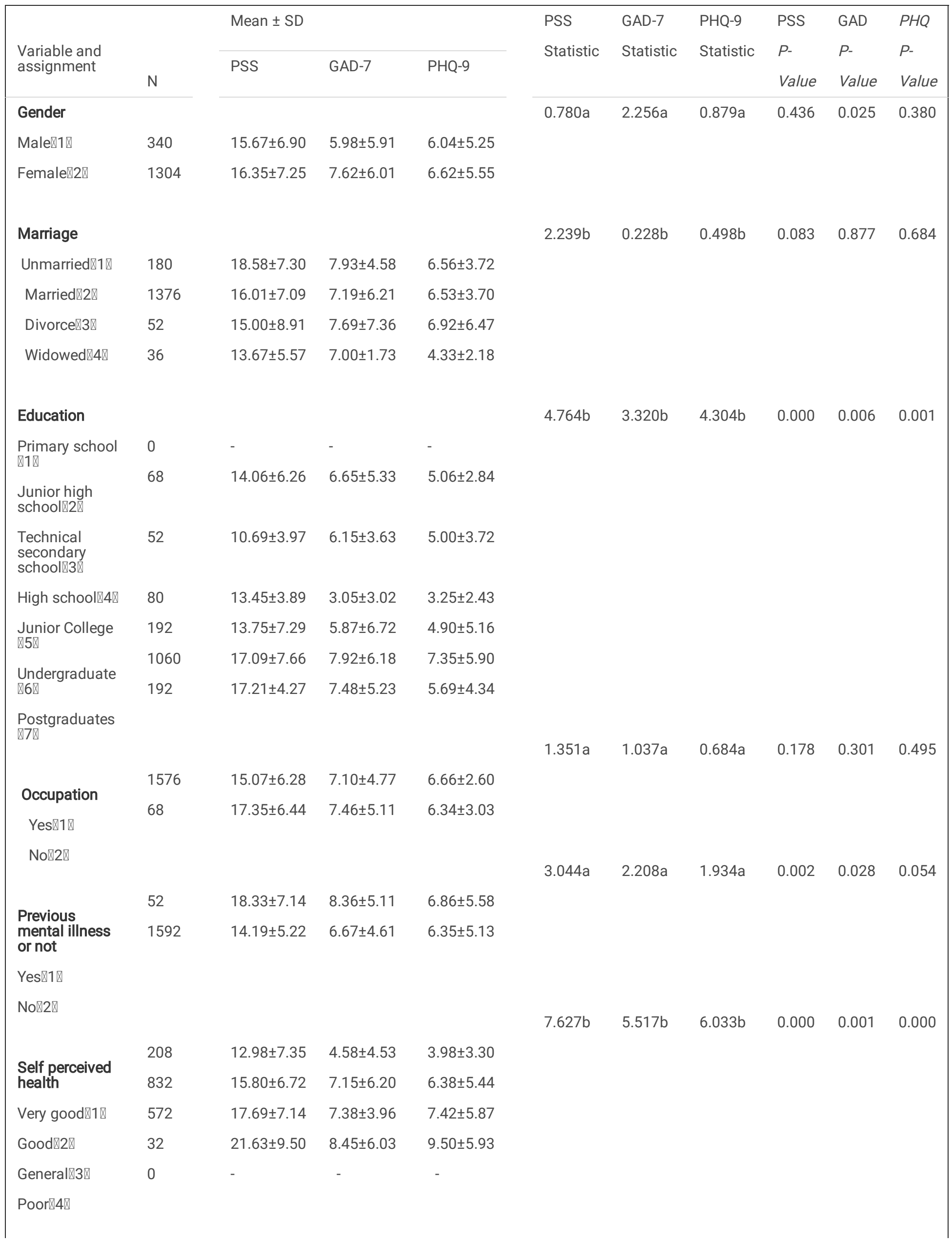


Very poor $₫ 5 \rrbracket$

$\begin{array}{llllll}2.664 a & 6.152 a & 2.644 a & 0.041 & 0.001 & 0.041\end{array}$

$\begin{array}{lllll}\begin{array}{l}\text { COVID-19 } \\ \text { infection or not }\end{array} & 24 & 21.53 \pm 4.38 & 13.30 \pm 2.19 & 9.50 \pm 2.74 \\ \text { Yes } ₫ 1 \rrbracket & 1620 & 16.14 \pm 7.19 & 7.20 \pm 6.02 & 6.46 \pm 5.51\end{array}$

No $₫ 2 \otimes$

\begin{tabular}{|c|c|c|c|c|c|c|c|c|c|c|}
\hline & 424 & $18.64 \pm 6.65$ & $8.98 \pm 5.61$ & $8.20 \pm 5.42$ & $4.126 a$ & $3.416 a$ & $3.754 a$ & 0.000 & 0.001 & 0.000 \\
\hline Yes $₫ 1 \rrbracket$ & 1220 & $15.37 \pm 7.17$ & $6.69 \pm 6.05$ & $5.91 \pm 5.40$ & & & & & & \\
\hline
\end{tabular}

No $₫ 2 \otimes$

Is the family

infected with

the COVID-19

Yes $₫ 1 \rrbracket$

No $₫ 2 \otimes$

$\begin{array}{llll}148 & 19.32 \pm 5.39 & 8.81 \pm 5.62 & 8.14 \pm 5.08 \\ 1496 & 15.90 \pm 7.26 & 7.13 \pm 6.05 & 6.34 \pm 5.51\end{array}$

Note: a represents $\mathrm{t}$ value, $\mathrm{b}$ represents $\mathrm{F}$ value

Table 4. Single factor analysis of total scores of PSS, GAD-7 and PHQ-9 scales of participants outside Hubei Province 


\begin{tabular}{|c|c|c|c|c|c|c|c|c|c|c|}
\hline \multirow{2}{*}{$\begin{array}{l}\text { Variable and } \\
\text { assignment }\end{array}$} & \multirow[b]{2}{*}{$\mathrm{N}$} & \multicolumn{3}{|l|}{ Mean \pm SD } & \multirow{2}{*}{$\begin{array}{l}\text { PSS } \\
\text { Statistic }\end{array}$} & \multirow{2}{*}{$\begin{array}{l}\text { GAD-7 } \\
\text { Statistic }\end{array}$} & \multirow{2}{*}{$\begin{array}{l}\text { PHQ-9 } \\
\text { Statistic }\end{array}$} & \multirow{2}{*}{$\begin{array}{l}\text { PSS } \\
P- \\
\text { Value }\end{array}$} & \multirow{2}{*}{$\begin{array}{l}\text { GAD } \\
P \text { - } \\
\text { Value }\end{array}$} & \multirow{2}{*}{$\begin{array}{l}\text { PHQ } \\
P \text { - } \\
\text { Value }\end{array}$} \\
\hline & & PSS & GAD- & $P H Q-9$ & & & & & & \\
\hline Gender & & & & & $1.683 a$ & $0.418 a$ & $0.720 a$ & 0.093 & 0.676 & 0.472 \\
\hline Male邓1》 & 540 & $13.49 \pm 7.11$ & $4.86 \pm 5.46$ & $5.32 \pm 6.19$ & & & & & & \\
\hline Female『2】 & 1116 & $14.73 \pm 6.91$ & $5.08 \pm 4.90$ & $5.77 \pm 5.82$ & & & & & & \\
\hline Marriage & & & & & $4.134 b$ & $1.282 \mathrm{~b}$ & $2.495 \mathrm{~b}$ & 0.007 & 0.280 & 0.059 \\
\hline Unmarried $₫ 1 \rrbracket$ & 556 & $15.85 \pm 7.29$ & $5.45 \pm 5.65$ & $6.68 \pm 6.55$ & & & & & & \\
\hline Married $\llbracket 2 \rrbracket$ & 1020 & $13.71 \pm 6.72$ & $4.91 \pm 4.79$ & $5.13 \pm 5.53$ & & & & & & \\
\hline Divorce $₫ 3 \rrbracket$ & 76 & $11.32 \pm 6.57$ & $3.47 \pm 4.23$ & $4.79 \pm 5.83$ & & & & & & \\
\hline Widowed $₫ 4 \rrbracket$ & 4 & 16.00 & 5.00 & 5.00 & & & & & & \\
\hline Education & & & & & $0.919 \mathrm{~b}$ & $1.454 b$ & $0.894 \mathrm{~b}$ & 0.481 & 0.193 & 0.499 \\
\hline Primary & 20 & $12.80 \pm 7.46$ & $5.20 \pm 7.33$ & $5.20 \pm 9.95$ & & & & & & \\
\hline $\begin{array}{l}\text { Junior high } \\
\text { school(2】 }\end{array}$ & 124 & $15.61 \pm 6.93$ & $6.87 \pm 6.35$ & $5.97 \pm 7.94$ & & & & & & \\
\hline Technical & 52 & $16.07 \pm 9.15$ & $6.69 \pm 6.02$ & $6.85 \pm 7.56$ & & & & & & \\
\hline secondary & 128 & $15.91 \pm 7.24$ & $6.09 \pm 5.24$ & $7.50 \pm 7.32$ & & & & & & \\
\hline 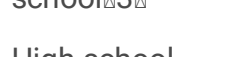 & 444 & $14.16 \pm 6.34$ & $4.68 \pm 4.86$ & $5.12 \pm 5.38$ & & & & & & \\
\hline 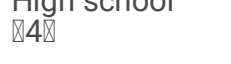 & 724 & $13.71 \pm 7.39$ & $4.70 \pm 4.95$ & $5.64 \pm 5.60$ & & & & & & \\
\hline 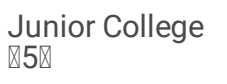 & 164 & $14.93 \pm 5.88$ & $4.46 \pm 4.25$ & $4.85 \pm 4.79$ & & & & & & \\
\hline $\begin{array}{l}\text { Undergraduate } \\
\bigotimes 6 \rrbracket\end{array}$ & & & & & $0.684 a$ & $1.338 a$ & $0.391 a$ & 0.495 & 0.182 & 0.697 \\
\hline Postgraduates & 1552 & $13.07 \pm 6.28$ & $4.90 \pm 4.77$ & $5.46 \pm 2.60$ & & & & & & \\
\hline & 104 & $14.35 \pm 6.44$ & $5.23 \pm 5.11$ & $5.79 \pm 3.03$ & & & & & & \\
\hline Occupation & & & & & & & & & & \\
\hline Yes冈1区 & & & & & $0.158 a$ & $0.381 \mathrm{a}$ & $1.271 \mathrm{a}$ & 0.875 & 0.703 & 0.205 \\
\hline No®2】 & 68 & $14.59 \pm 6.04$ & $5.47 \pm 3.69$ & $7.41 \pm 4.68$ & & & & & & \\
\hline $\begin{array}{l}\text { Previous } \\
\text { mental }\end{array}$ & 1588 & $14.31 \pm 7.04$ & $4.99 \pm 5.14$ & $5.54 \pm 5.98$ & & & & & & \\
\hline illness or not & & & & & $7.248 b$ & $10.953 b$ & $9.595 b$ & 0.000 & 0.000 & 0.000 \\
\hline Yes $\triangle 1 \otimes$ & 304 & $12.08 \pm 5.63$ & $3.49 \pm 4.48$ & $3.87 \pm 4.70$ & & & & & & \\
\hline No®2】 & 764 & $13.97 \pm 6.40$ & $4.53 \pm 4.47$ & $4.94 \pm 5.18$ & & & & & & \\
\hline & 540 & $15.40 \pm 7.66$ & $5.87 \pm 5.39$ & $6.92 \pm 6.59$ & & & & & & \\
\hline Self perceived & 40 & $21.40 \pm 9.56$ & $11.70 \pm 6.63$ & $11.90 \pm 8.03$ & & & & & & \\
\hline 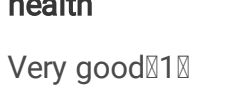 & 8 & $26.00 \pm 2.83$ & $16.50 \pm 6.36$ & $18.0 \pm 12.73$ & & & & & & \\
\hline Good $₫ 2 \rrbracket$ & & & & & 1776 & & & & & 110 \\
\hline General『3》 & & & & & $1.376 a$ & $2.040 \mathrm{a}$ & $0.825 a$ & 0.169 & 0.042 & 0.410 \\
\hline
\end{tabular}




\begin{tabular}{|c|c|c|c|c|c|c|c|c|c|c|}
\hline \multirow[t]{2}{*}{ Very poor $₫ 5 \rrbracket$} & 20 & $18.60 \pm 5.81$ & $9.60 \pm 5.73$ & $7.80 \pm 6.14$ & & & & & & \\
\hline & 1636 & $14.27 \pm 6.70$ & $4.95 \pm 5.06$ & $5.59 \pm 5.94$ & & & & & & \\
\hline \multicolumn{11}{|l|}{$\begin{array}{l}\text { COVID-19 } \\
\text { infection }\end{array}$} \\
\hline or not & & & & & $2.477 a$ & $2.761 a$ & $2.980 a$ & 0.014 & 0.006 & 0.003 \\
\hline Yes $₫ 1 \rrbracket$ & 164 & $16.88 \pm 7.63$ & $7.07 \pm 5.68$ & $8.22 \pm 6.84$ & & & & & & \\
\hline & 1492 & $14.05 \pm 6.87$ & $4.78 \pm 4.97$ & $5.34 \pm 5.77$ & & & & & & \\
\hline \multicolumn{11}{|l|}{$\begin{array}{l}\text { Isolation or } \\
\text { not }\end{array}$} \\
\hline Yes $\triangle 10$ & 20 & $18.60 \pm 5.81$ & $9.60 \pm 5.73$ & $7.80 \pm 6.14$ & & & & & & \\
\hline $\mathrm{No} \otimes 2 \bigotimes$ & 1636 & $14.27 \pm 6.70$ & $4.95 \pm 5.06$ & $5.59 \pm 5.94$ & & & & & & \\
\hline \multicolumn{11}{|l|}{$\begin{array}{l}\text { Is the family } \\
\text { infected with } \\
\text { the COVID-19 }\end{array}$} \\
\hline \multicolumn{11}{|l|}{ Yes $\otimes 10$} \\
\hline No®2】 & & & & & & & & & & \\
\hline
\end{tabular}

Note: a represents $t$ value, $b$ represents $F$ value

Table 5. Multiple stepwise regression analysis of influencing factors of total scores of PSS, GAD-7, and PHQ-9 in Hubei Province 


\begin{tabular}{|lllllll|}
\hline Scale & Items & B & Standard error of B & Standardized B & T-value & P-value \\
\hline PSS & Constant & 22.463 & 7.958 & - & 2.823 & 0.005 \\
& Educational level & 1.004 & 0.303 & 0.156 & 3.316 & 0.001 \\
& Mental illness or not & -8.518 & 3.093 & -0.130 & -2.754 & 0.006 \\
& Physical health & 2.348 & 0.481 & 0.228 & 4.878 & 0.000 \\
& CoVID-19 infection & -3.281 & 1.135 & -0.131 & -2.891 & 0.004 \\
& Isolation or not & -2.506 & 1.173 & -0.100 & -2.136 & 0.033 \\
& Is the family infected with COVID-19 & -2.363 & 0.764 & -0.144 & -3.093 & 0.002 \\
& & & & & & \\
& & & & & & \\
GAD-7 & Constant & 14.266 & 6.875 & - & 2.075 & 0.039 \\
& Gender & 1.924 & 0.718 & 0.130 & 2.680 & 0.008 \\
& Mental illness or not & -5.948 & 2.660 & -0.108 & -2.236 & 0.026 \\
& Physical health & 1.533 & 0.414 & 0.177 & 3.703 & 0.000 \\
CovID-19 infection & -5.507 & 2.378 & -0.101 & -2.127 & 0.034 \\
Isolation or not & -2.068 & 0.672 & -0.150 & -3.080 & 0.002 \\
Constant & 4.609 & 5.510 & - & 0.836 & 0.403 \\
Education level & 0.571 & 0.235 & 0.116 & 2.430 & 0.016 \\
Physical health & 1.497 & 0.377 & 0.190 & 3.972 & 0.000 \\
Isolation or not & -1.833 & 0.604 & -0.146 & -3.035 & 0.003 \\
\hline
\end{tabular}

Table 6. Multiple stepwise regression analysis of influencing factors of total scores of PSS, GAD-7, and PHQ-9 of participants outside Hubei Province 


\begin{tabular}{|c|c|c|c|c|c|c|}
\hline Scale & Items & B & Standard error of B & Standardized B & T-value & P-value \\
\hline \multirow[t]{5}{*}{ PSS } & Constant & 17.825 & 2.511 & - & & 0.000 \\
\hline & Marriage & -2.092 & 0.590 & -0.167 & 7.100 & 0.000 \\
\hline & Physical health & 2.096 & 0.422 & 0.234 & -3.544 & 0.000 \\
\hline & Isolation or not & -2.385 & 1.101 & -0.102 & 4.970 & 0.031 \\
\hline & & & & & -2.165 & \\
\hline \multirow[t]{4}{*}{ GAD-7 } & Constant & 9.484 & 4.509 & - & 2.103 & 0.036 \\
\hline & Physical health & 1.669 & 0.308 & 0.256 & 5.412 & 0.000 \\
\hline & Isolation or not & -1.863 & 0.830 & -0.110 & -2.244 & 0.025 \\
\hline & Family infected with COVID-19 & -3.286 & 1.352 & -0.071 & -2.430 & 0.016 \\
\hline \multirow[t]{4}{*}{ PHQ-9 } & Constant & 6.289 & 2.002 & & 3.142 & 0.002 \\
\hline & Physical health & 1.972 & 0.359 & - & 5.497 & 0.000 \\
\hline & Isolation or not & -2.640 & 0.936 & 0.259 & -2.819 & 0.005 \\
\hline & & & & -0.133 & & \\
\hline
\end{tabular}

Figures 


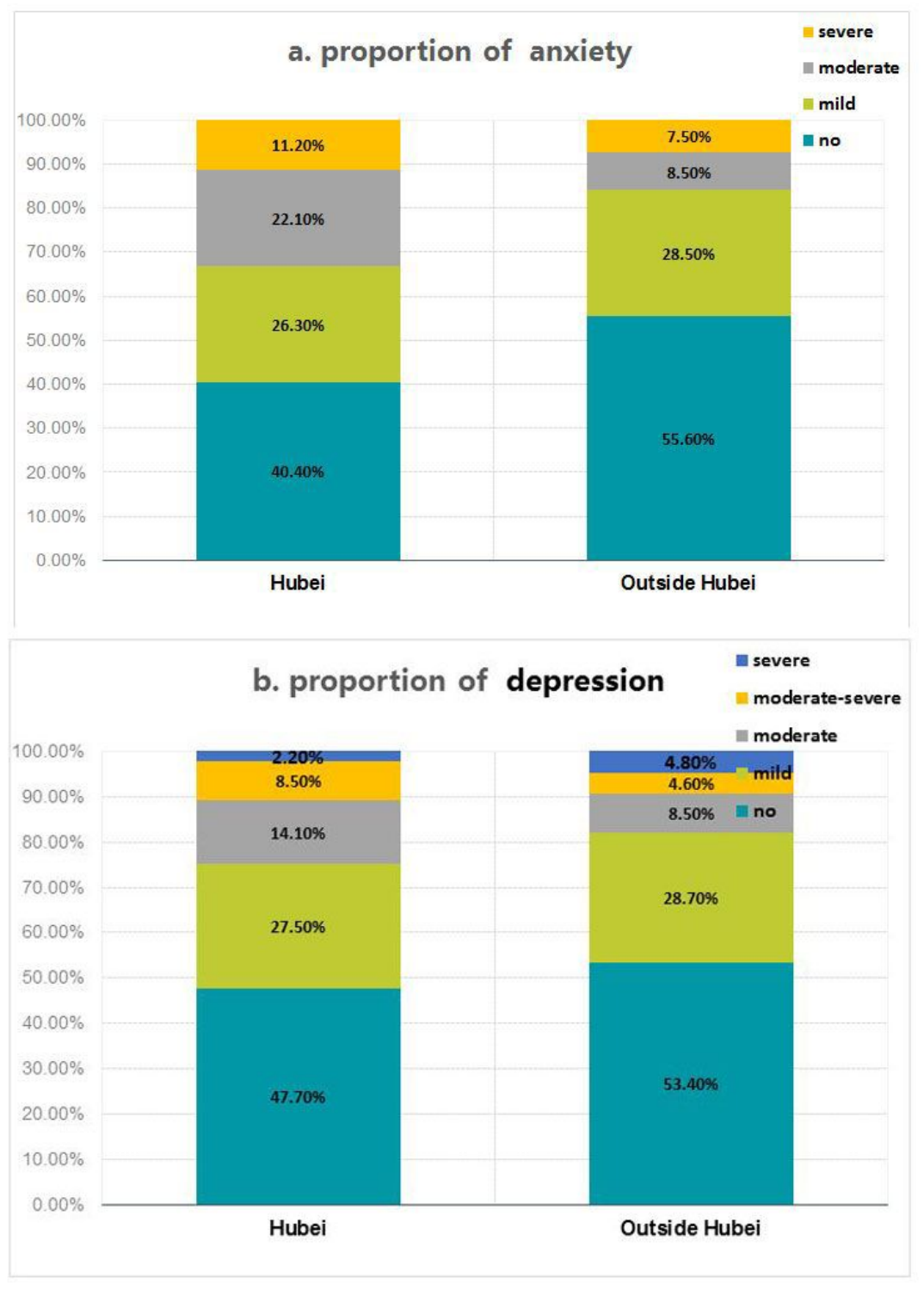

Figure 1

The different proportions of anxiety and depression between subjects from and outside Hubei. 